Summary: A theoretical model describing aggregation of filler particles in amorphous elastomers is proposed. The model is based on a counting technique originally used in genome analysis to characterize the size and distribution of overlapping segments randomly placed on a DNA molecule. In the present model, the particles are first assumed to aggregate randomly upon mixing into the elastomer and their sizes are calculated. The sizes and distributions of aggregates are also studied in the presence of attractive interparticle forces. Results of the proposed model are compared with experimental data on silica-filled end-linked poly(dimethylsiloxane) networks. Comparison of the theory and experiment shows that the random aggregation assumption where no attractive forces exist between the particles is not valid and a significant attraction between the silica particles is needed in the theory to justify the experimental data obtained using atomic force microscopy. For filler content below 1.45 vol.$\%$, the model agrees, qualitatively, with experiment and shows the increase in cluster size with increasing amount of filler. It also explains the increase in the dispersion of aggregate sizes with increasing amount of filler.

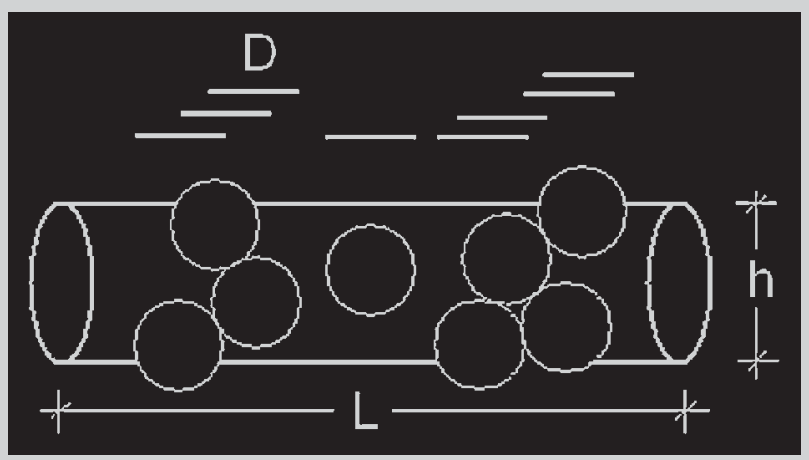

Clustering of the primary silica particles in an imaginary volume of poly(dimethylsiloxane) network.

\title{
Aggregation of Fillers Blended into Random Elastomeric Networks: Theory and Comparison with Experiments
}

\author{
Mustafa M. Demir, ${ }^{* 1, a}$ Yusuf Z. Menceloglu, ${ }^{1}$ Burak Erman ${ }^{2}$ \\ ${ }^{1}$ Faculty of Engineering and Natural Sciences, Sabanci University, Orhanli 34956 Istanbul, Turkey \\ ${ }^{2}$ Department of Chemical and Biological Engineering, Koc University, Rumelifeneri yolu, Sariyer 34450, Istanbul, Turkey \\ E-mail: demir@mpip-mainz.mpg.de
}

Received: April 17, 2006; Revised: June 16, 2006; Accepted: June 22, 2006; DOI: 10.1002/macp.200600185

Keywords: aggregation; fillers; nanoparticles; poly(dimethylsiloxane); silica

\section{Introduction}

Significant aggregation takes place when nanoparticles are blended into a random amorphous polymeric medium, contrary to in situ generation which results in uniform size distributions. ${ }^{[1]}$ In the present paper, we propose a theoretical model to study the size and distribution of aggregates that take place when the particles are blended into a random amorphous mobile elastomeric medium. We test the predictions of the model with experimental results on silica-filled end-linked poly(dimethylsiloxane) (PDMS) networks and explain the increase in the degree of aggregation with increase in filler content.

\footnotetext{
a Present address: Max Planck Institute for Polymer Research, D55021 Mainz, Postfach 3148, Germany. Fax: +496131379330.
}

Organic/inorganic nanocomposites with different levels of combinations have important applications in optics, ${ }^{[2]}$ electronics, ${ }^{[3]}$ and rubber elasticity. ${ }^{[4]}$ The efficiency of the nanocomposites depends on dispersion, size distribution of the guest inorganic particles in host organic matrix, and their specific interactions with polymer chains. The homogenous dispersion of uniform-sized nanoparticles is the most desired characteristic of these materials. However, clustering or aggregation of inorganic nanoparticles is often a problem, especially when the particles are incorporated into a matrix by blending rather than in situ generation (throughout the paper we use the terms cluster and aggregate interchangeably). The smaller (non-aggregated) particles provide larger contact area between the organic and inorganic components due to large surface-to-volume ratio 
and are desirable for many applications. Despite the importance of the problem of aggregation of particles in composites, a detailed model explaining the role of different factors in such systems does not yet exist.

PDMS/silica is one of the most popular pairs of these nanocomposite materials. Silica is a strong and efficient reinforcing agent of PDMS ${ }^{[5]}$ and perfect filler due to the ease of surface modification. ${ }^{[6]}$ Strong polymer-filler interactions are responsible for the improvement of mechanical properties of silicone rubber. These interactions lead to the adsorption of polymer molecules on the filler's surface and to the formation of a thin layer of "bound rubber" whose physical and chemical properties are different from bulk rubber. ${ }^{[7,8]}$ The molecular structure of the bound rubber and its effect on the network properties has been investigated experimentally by means of DSC ${ }^{[7]}$ and ${ }^{1} \mathrm{H}$ NMR spectroscopy. ${ }^{[7,9-11]}$ High resolution NMR spectroscopy shows that physically adsorbed PDMS chains are immobilized at the silica surface with a small fraction of monomer $\mathrm{Si}\left(\mathrm{CH}_{3}\right)_{2} \mathrm{O}$ - units. The remaining portions of the chains situated outside the interface are mobile. The immobilized chains cause a substantial decrease in the heat capacity at the glass transition temperature. ${ }^{[7]}$ Gussoni et al. ${ }^{[11]}$ also used NMR spectroscopy to investigate silica-filled PDMS rubbers, and observed decrease in segmental mobility of the PDMS chains in the vicinity of the filler surface. Berriot et al. ${ }^{[9]}$ employed ${ }^{1} \mathrm{H}$ NMR experiments on filled rubbers and observed a layer of immobilized segments at the particle surface. In the case of chains chemically grafted to the silica surface, they observed immobile layers which they described as a glassy shell around the filler surface. Cosgrove et al. ${ }^{[12]}$ used DSC and NMR measurements, and showed that the reduction in the mobility of the PDMS chains corresponded to a shift in the glass transition to higher temperatures. These shifts are relatively small; nevertheless, they indicate the presence of a gradient of segmental mobility in going from the rubber towards the silica surface. Although the reinforcement mechanism of PDMS is not completely understood yet, it is well-known that the polymer-filler interactions control the macroscopic mechanical behavior of nanocomposite materials. The extent of reinforcement, i.e., the extent of adsorption, is a function of adhesion forces between polymer and filler, the surface area of the filler, molecular weight of the polymer, and concentration of the filler. ${ }^{[5,8]}$ The quantity of polymer adsorbed per unit weight of silica is found to be independent of the filler concentration, assuming perfect wetting of the silica surface. However, it is proportional to the number of silanol groups at the silica surface. The thickness of the polymer layer around the silica particles, known as bound rubber, is in the order of the size of a polymer molecule, i.e., $1-2 \mathrm{~nm} .{ }^{[7]}$ Bound rubber cannot be extracted from uncured silicone rubber completely. According to results of Aranguren, the percentage of the chains situated at the interface are 5.3 and 11.8 when the silica fractions are 8 and 15 vol.-
$\%$, respectively. (The surface area of filler they used was $108 \mathrm{~m}^{2} \cdot \mathrm{g}^{-1}$. $)^{[8]}$ The kinetics of adsorption was studied by Levresse et al. ${ }^{[13]}$ Rate constant of adsorption was found to depend on the strength of the interaction between the chain ends, the silanol groups on the particles, and the molecular weight of the chains. ${ }^{[14-16]}$ However, the rate was independent of the silica content. ${ }^{[8]}$ Dynamical rheological properties of uncured dispersion of silica in liquid polyisoprene (PI) and PDMS-PI block copolymer were investigated by Gurovich et al. ${ }^{[17,18]}$ They observed an increase in dynamic storage modulus $\left(G^{\prime}\right)$ with decreasing PI molecular weight and with increasing silica content. They also claimed that the amount of bound rubber as well as the fraction of PDMS segments on the block copolymer play an important role on the mechanical properties of the silica/ PDMS-PI solution.

Silica powder consists of aggregates of individual spherical particles, which are fused together, where each particle is $10-20 \mathrm{~nm}$ in diameter. Agglomerates are clusters of aggregates, which are assumed to be the primary structures in nanocomposite systems, connected by secondary forces such as hydrogen bonding and van der Waals interactions. ${ }^{[8]}$ Scattering techniques have been used in the characterization of colloidal particles. ${ }^{[19]}$ The size of the colloids in suspension has been controlled either by adding salt or adjusting the $\mathrm{pH}$ of the solution. ${ }^{[20]}$ Simovic and Prestidg $\mathrm{e}^{[21]}$ investigated the adsorption and subsequent aggregation of silica particles on the PDMS droplet-water interface. The packing behavior of primary particles in small clusters, which are attached to the droplet surface, is discussed in detail by Manoharan et al. ${ }^{[22]}$ Size control is governed by particle-droplet and particle-particle interactions through colloidal forces. As can be seen from these examples, all of the work in this field focused on the agglomeration phenomenon of silica in colloidal solutions, and only a small amount of information is present on agglomeration in the bulk. In contrast to silica, a detailed study was performed on fractal structures of carbon black in rubbers by Klüppel et al. ${ }^{[23]}$ The mechanisms of cluster growth at carbon black concentrations below and above gel point were clarified. They further investigated the effect of filler networking on mechanical and electrical properties of elastomers on the basis of percolation theory and kinetic cluster-cluster aggregation model, respectively. ${ }^{[24]}$

The size of silica filler in an organic matrix depends on the methods by which the particles are produced. Silica can be introduced into the matrix by either blending with polymer solution (melt) prior to crosslinking or by adding additional tetraethoxysilane (TEOS) for in situ silica precipitation through the sol-gel reaction. In situ precipitation is an efficient technique for producing almost monodisperse particles with no or negligible agglomeration. ${ }^{[1,25]}$ Agglomeration problem is important in blending, however. Aranguren et al. ${ }^{[8]}$ studied the agglomeration in mechanically mixed suspensions of silica in PDMS. Yuan 
and Mark $^{[5]}$ compared the results of the two different techniques.

Despite a large body of work in this field, as reviewed in the preceding paragraphs, a theoretical model that systematically explains the dependence of the extent of aggregation on filler content in blended systems is still lacking. In the present study, we propose a statistical model that establishes a quantitative relationship among filler content, the strength of the surface forces, and the resulting distribution of aggregate sizes. We compare the predictions of the model with experimental data obtained from end-linked PDMS networks into which silica particles are blended. The experimental work and the proposed model are presented in the following two sections, respectively. In the section Comparison with Experimental Results, predictions of the model are compared with results of atomic force microscope (AFM) measurements on the silica particles. In section 5, we discuss the merits and the limitations of the model. The mathematical details of the model are presented in the Appendix.

\section{Experimental Part}

The fumed silica (CAB-O-SIL-S5505), which has $225 \mathrm{~m}^{2} \cdot \mathrm{g}^{-1}$ surface area and $14 \mathrm{~nm}$ of non-aggregated single particle diameter, was used without any surface treatment. Hydroxyl groups cover $40 \%$ of the silica surface. Suspensions of untreated fumed silica in PDMS/toluene solution $\left(10 \mathrm{~g} \cdot(20 \mathrm{~g})^{-1}\right)$ were prepared at room temperature and mixed with a stirrer for $2 \mathrm{~h}$ followed by sonicating for $2 \mathrm{~min}$ to destroy agglomeration. The effect of stirring time was checked by preparing samples by stirring for $24 \mathrm{~h}$. The results did not show significant differences compared those obtained by stirring for $2 \mathrm{~h}$. The hydroxyl terminated PDMS (Baysilone oil T 50-GE Bayer Silicone) was end-linked into a network by reacting with an excess amount of the crosslinking agent, TEOS. The filler content varied in the range of $0-2.45$ vol.- $\%$. TEOS (600 $\mu$ l Sigma) and the catalyst, tin(II) 2-ethylhexanoate (Aldrich), were added to the dispersion while stirring. The end-linking reactions were carried out in a Teflon mold at room temperature for $24 \mathrm{~h}$ and at $80^{\circ} \mathrm{C}$ overnight. The thickness of the resulting film was in the order of $2 \mathrm{~mm}$. The molecular weight of PDMS is $53 \mathrm{kDa}\left(n_{\mathrm{w}}=675\right)$, where $n_{\mathrm{w}}$ is the weight-average degree of polymerization. The polydispersity index was close to 1.62 .

Although the level of aggregation in the network is independent of stirring time, the nanoparticles come already aggregated before introducing into the polymer solution, and hence the stated diameter of $14 \mathrm{~nm}$ is that of a non-aggregated single particle. Elimination of the aggregates and the separation into individual particles cannot be achieved unless the particles have an efficient surface modification. Ultrasonication is applied prior to end-linking to minimize the aggregation of the silica particles. It has to be noted that ultrasonication, which employs a sound wave, is efficient when the size of the aggregate is comparable with that of the wavelength. The aggregates smaller than the wavelength are not separated as efficient as the particles having comparable size with the wavelength. Thus, aggregation is minimized but not eliminated completely at the beginning of the composite synthesis. In the present study, the "individual filler particle size" is defined as the average size of the aggregate obtained after sonication.

The surface of PDMS/silica films was imaged by tappingmode AFM (Nanoscope IIIa, Digital Instruments) using oxidesharpened Si tips, and silica particles were detected on the phase images by comparing them with the heighted ones. The dimensions and size distributions of silica clusters were determined by particle analysis option of the AFM Nanoscope software. A source of uncertainty might be expected in two-dimensional AFM imaging. Particles overlapping in one dimension may appear as one cluster even though they are not parts of the same aggregate. In fact, this argument is ruled out if the thin depth of field and the high resolution of AFM are considered. In tapping mode of AFM imaging, the depth of field, i.e., the information one can get, is limited with the outermost layer or the layer below the surface of the specimen. In other words, AFM is not able to give information deeper than its depth of field and, therefore overlapping taking place out of the surface layer (in the bulk) cannot be seen. The occurrence of this overlapping in the interaction volume between the AFM tip and the surface of specimen at low particle concentrations (volume fractions are lower than 0.025) is unlikely. Even if the overlapping occurs in the surface layer, the resolution of the AFM is powerful enough to figure out the real aggregate structure (lateral: $\approx 1 \mathrm{~nm}$ and vertical: $\approx 0.1 \mathrm{~nm}$ ).

\section{Theory and the Model}

In this section and in the Appendix, we present a theoretical model that leads to the determination of the distribution of aggregate size, and therefore to the mean aggregate size and their dependence on filler content. As stated in the Introduction, we use the terms cluster and aggregate interchangeably. The model is based on the following three basic assumptions:

(1) The individual particles are spherical and of uniform size with diameter $D$.

(2) The individual particles are assumed to be randomly placed within the volume of the material. The aggregation of the randomly deposited particles results from fluctuations in the density of particles in the system as a result of which several particles may touch each other and form clusters as the amount of particles increases. The formation of a cluster is defined here as "random aggregation". The randomness assumption implicitly states that there are no attractive forces between the individual particles, and the only interaction is that of excluded volume, where two particles cannot penetrate each other.

(3) The matrix surrounding a given particle forms a sufficiently mobile environment. This assumption is made to ensure that if two or more particles are to meet randomly while mixing the filler and the matrix, they should not be hindered from doing so due to the 
presence of the matrix. The main consequence of this assumption is that the polymer does not significantly change the particle size distribution of the filler. In the experiments, the choice of a PDMS matrix in the presence of a diluent provides the mobile environment stated in this assumption. A thermoplastic matrix, for example, would be too rigid and fails to provide a sufficiently mobile environment. This assumption is supported by the experimental work of Aranguren et al. ${ }^{[8]}$ where the size distribution of silica particles were determined before and after mixing with different molecular weights of PDMS chains. Two effects of mixing with PDMS were observed. First, there was a significant decrease in the aggregate size, which was attributed to the breakup of particles due to mixing. Second, there was a small narrowing of the distribution of particle sizes. In other words, no significant distortion of the particle size distribution was observed which is in support of our assumption that the presence of the polymer does not change the distribution significantly.

For low degrees of filler content, assumption 2 leads to insignificant degrees of aggregation, contrary to experimental data. For this reason we modify assumption 2 and consider the following case:

$\left(2^{\prime}\right)$ The particles interact favorably with each other, and therefore form clusters which are larger than those obtained in the random case.

The model is based on counting the number of particles and clusters in a cylindrical volume. In Figure 1, a small cylindrical volume is depicted in which there are eight individual particles each of diameter $D$ that form three clusters with one, three, and four particles. We do not elaborate on the specific dimensions of the cylinder, except that (i) its length, $L$, should be sufficiently large so that the number of individual particles in the cylinder is large and (ii) its diameter, $h$, should be in the order of average cluster size so that a given cross-section contains a single cluster, on the average. In the calculations that are presented in full detail in the Appendix, we take the diameter of the cylinder to be equal to the average cluster size, $\bar{D}_{\mathrm{c}}$. Also shown in Figure 1 are the projections of the diameters of the particles

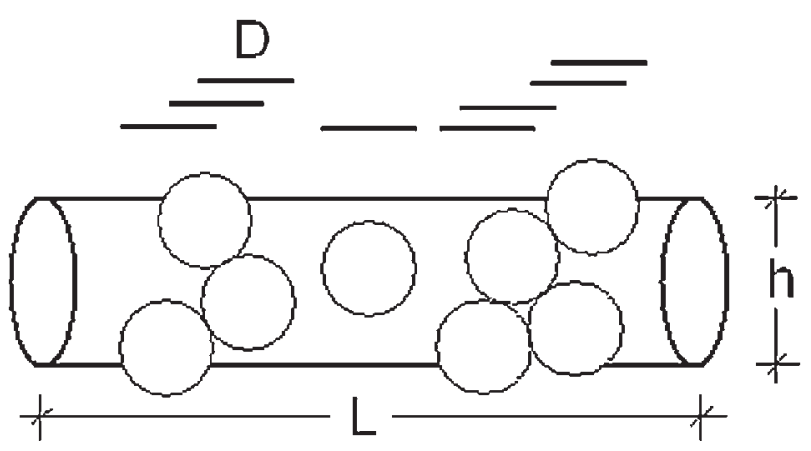

Figure 1. Clusters of individual particles in a small cylinder. on an axis parallel to the axis of the cylinder. Each projection is a line segment of length $D$. If two particles are in contact, their projections on the axis overlap, as can be observed from Figure 1. Thus, a cluster of particles is represented by a group of overlapping projection lines. The projections defined in this manner allow for a counting scheme that allows us to determine the cluster formation probabilities and cluster sizes of the filler particles, and finally to the distribution of cluster sizes.

Mapping the three-dimensional clusters into their onedimensional projections as described above is an approximation that simplifies the problem significantly. The statistical analysis of overlapping objects in one dimension has previously been used in great detail in the area of genome analysis. ${ }^{[26]}$ There, segments of fixed length are projected randomly on the long genome and the sizes of contiguous clusters of segments are calculated. In the present paper, as described in detail in the Appendix, we use the same mathematical model for the case of random filler aggregation. The details of statistical formulation of onedimensional cluster sizes and details of calculations are given in ref. ${ }^{[26]}$ and will not be repeated here for the interest of brevity. However, in order to reduce cross-referencing, the computational details are presented in the Appendix.

The theoretical model whose basic features are described above leads to the distribution of aggregate sizes as

$$
P(n)=\frac{\mathrm{e}^{-a_{0} e^{\alpha}}}{\sqrt{2 \pi}}\left[\frac{\mathrm{e}^{(\alpha+1) n}\left(\frac{a_{0}}{n}\right)^{n}}{\sqrt{n}}\right]
$$

This expression is derived in the Appendix and is given as Equation (A15). Here, $n$ is the number of individual silica particles in a cluster. The term "cluster size" is used synonymously with the number of individual silica particles in the cluster. $a_{0}$ is a parameter of the model [see Equation (A3) for its definition in terms of average cluster size], $\bar{D}_{\mathrm{c}}$, diameter of the individual filler particle, and the volume fraction, $v$, of fillers). The variable $\alpha$ is the coefficient that expresses the strength of the interfacial energy per unit area, $\gamma$, for a filler particle, and is defined as

$$
\alpha=\frac{4 \pi r^{2} \gamma}{k T}
$$

where, $r$ is the effective radius of a filler particle, $k$ the Boltzmann constant and $T$ the absolute temperature.

The average diameter, $\bar{D}_{\mathrm{c}}$, of a cluster is given according to the model as

$$
\frac{\bar{D}_{\mathrm{c}}}{D}=1+\frac{3}{4} \mathrm{e}^{\alpha} v
$$

where, $D$ is the diameter of the individual particle, and $\alpha$ and $v$ are as defined above.

Equation (1) and (3) are the main predictions of the model which will be compared with results of experiments in the following section. 

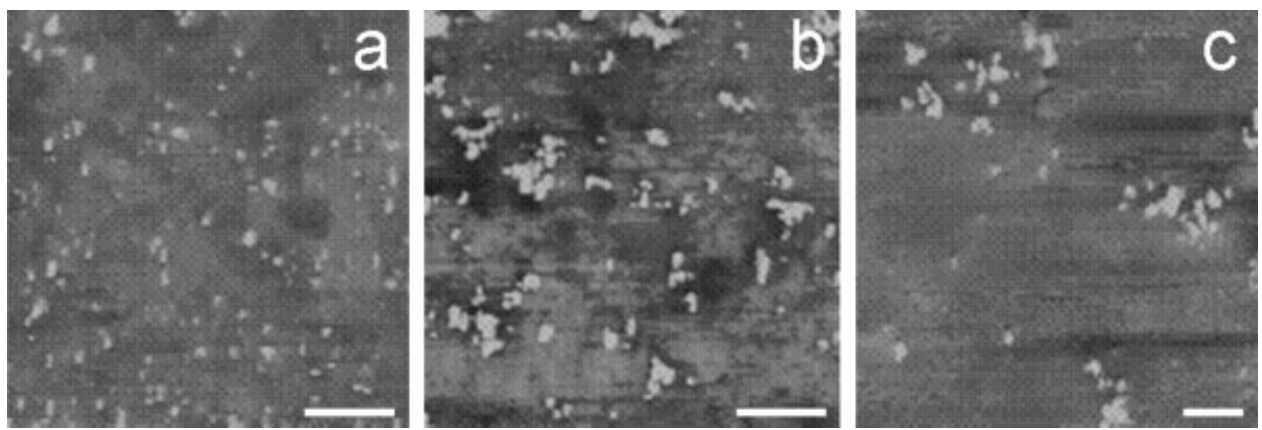

Figure 2. Tapping mode AFM micrographs of silica particles in PDMS matrix. The silica contents are for (a) 0.48 , (b) 1.45, and (c) 2.45 vol.- $\%$. The scale bars shown in the images are $1 \mu \mathrm{m}$.

\section{Comparison with Experimental Results}

In this section, we compare the predictions of the theoretical model with results of experiments on silica-filled PDMS networks. A total of six samples with silica volume percent of $0.24,0.48,0.96,1.45,1.95$, and 2.45 were prepared and tested. In Figure 2, sections for 0.48, 1.45, and 2.45 vol.-\% are presented. The cluster sizes show different degrees of distribution for different filler contents. In Figure 3, the distributions of cluster diameters are shown. Several interesting features are observed: increasing the filler content results in (i) larger average cluster diameters, (ii) decrease in the maxima of the distributions and increase in dispersion, and (iii) skewed distribution curves, showing a long tail extending to large cluster sizes. Mean cluster diameters are plotted as a function of filler content (not shown). A linear relationship is observed, where the straight line is the best fitting line with equation $\bar{D}_{\mathrm{c}}=D+m v$, with $D=36.7 \mathrm{~nm}$ and $m=9228$. Substituting these values into Equation (3) leads to a value of $\alpha=5.8$.

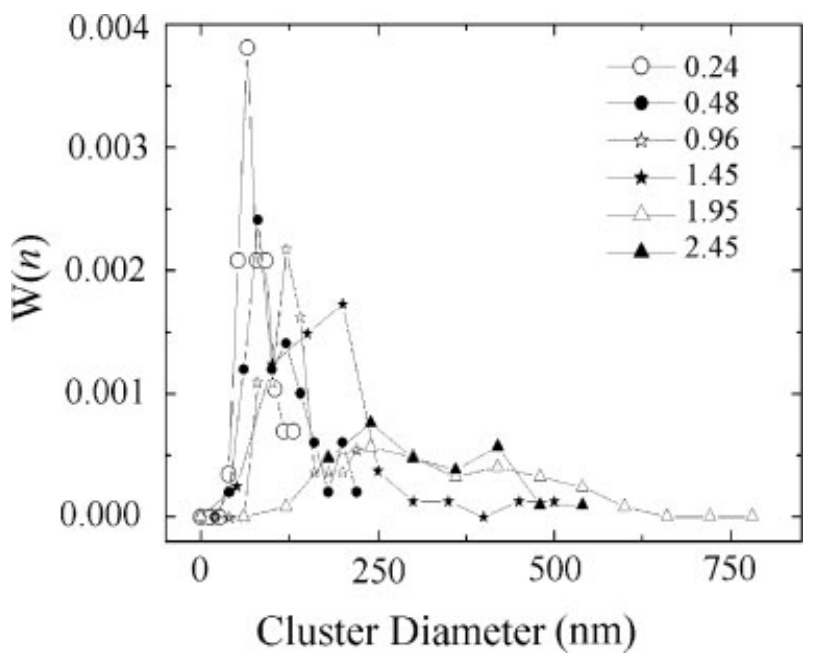

Figure 3. The distributions of the particle diameters prepared from different volume fractions of silica.
In Figure 4, results of Equation (1) are presented for $P(n)$ as a function of $n$. The five curves correspond to the five different filler contents used in our experiments, $v=0.0024$, $0.0048,0.0096,0.0145$, and 0.0195 . The filler content dependence enters Equation (1) through the parameter $a_{0}$. The fifth filler content used in the experiments, $v=0.0245$, was too high to allow accurate computation of the distribution $P(n)$ using Equation (1) and hence does not appear in Figure 4. The magnitudes of the maxima of the curves decrease with increasing filler content. Also, the dispersion, i.e., the spreading out of the curves, increases with increasing filler content. Both of these observations are in qualitative agreement with experimental data presented in Figure 2 and serve as the most significant test of the theory. Average values $\bar{n}$ of $n$ corresponding to each curve in Figure 4 are obtained using the expression $\bar{n}=\int_{1}^{\infty} n P(n) \mathrm{d} n$, where $P(n)$ is given by Equation (1). The corresponding $\overline{D_{\mathrm{c}}}$ values, obtained from $\bar{n}$ using the expression $\bar{D}_{\mathrm{c}}=D_{n}^{-1 / 3}$ for compact arrangement of particles, are plotted as a function of $v$ in Figure 5. The filled points are obtained from

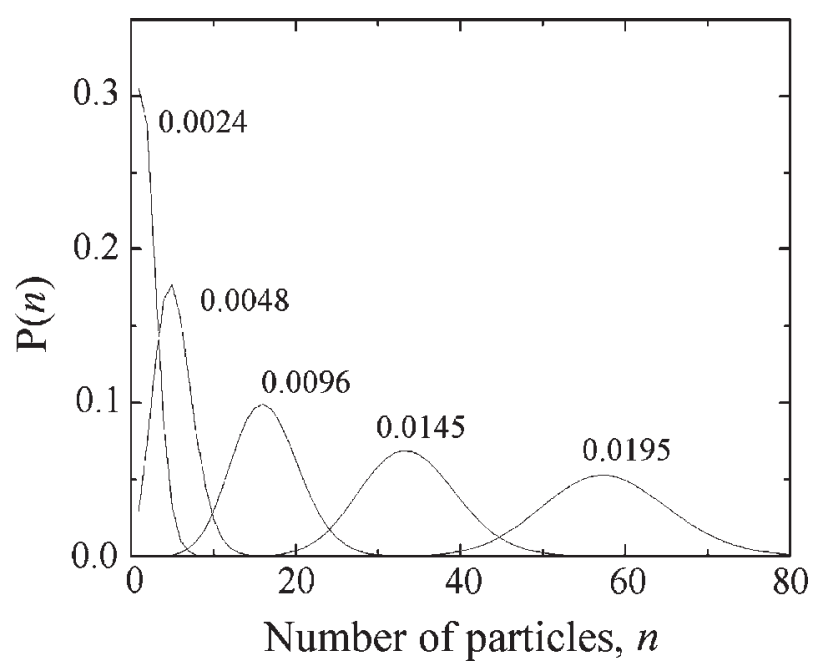

Figure 4. The distributions of particle diameters based on the results of the proposed model. 


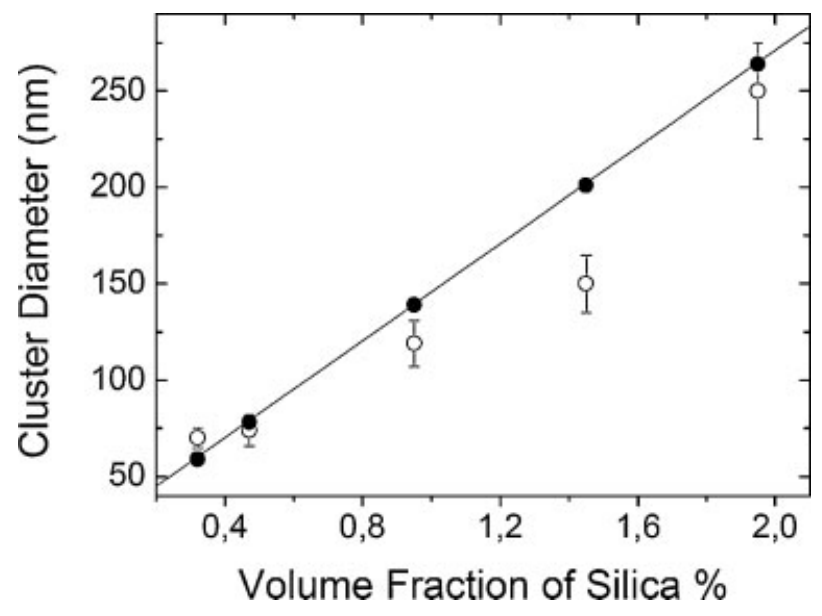

Figure 5. Comparison for measured and predicted cluster diameters at different filler contents in PDMS matrix, 0.24, 0.48, $0.96,1.45$, and 1.95 vol.- $\%$. Open circles and filled points refer to experimental and predicted values, respectively. The straight line is the best fit to the predicted points.

these calculations, and the line is the best fitting line. The open circles are experimental points, and fall close to the predicted values. This agreement may be accepted as a selfconsistency check of the formulation, and indicates that the distributions derived from the proposed model are accurate representations of filler aggregation.

\section{Concluding Remarks and Discussion}

The statistical model presented in this paper is based on the counting technique employed in contig size analysis in DNA technology. As discussed in presenting Figure 1 above, the three-dimensional particles are projected into one dimension for counting the state of overlap. We succeeded in formulating the aggregation problem only after adopting this simplifying assumption. One source of error in reducing the problem to a one-dimensional problem may be miscounting, where two aggregates may be neighboring but independent in the imaginary cylinder, and upon projection into one dimension they may be counted as a single cluster. An exact three-dimensional analysis that would remove these shortcomings would be prohibitively complex at this time. Assuming that aggregate size distributions are independent along three coordinate directions, one can approximate the three-dimensional distribution, $P(n)$, as $P(n)=P_{x}(n) P_{y}(n) P_{z}(n)$, where $P_{x}\left(\right.$ or $P_{y}$ or $\left.P_{z}\right)$ is the distribution along one dimension given by Equation (1). Then, the general shape of the three-dimensional distribution or the two-dimensional distribution that is observed in the actual AFM measurements will be similar to that given by Equation (1). The results based on this projection technique lead to results that were self-consistent, and also in agreement with the results of AFM measurements on silica-filled PDMS networks. However, the discussion pre- sented in this paragraph pertains to the similarities of the shapes of the distributions in one, two, or three dimensions. It should be noted that the problem itself is an aggregation problem in three dimensions and a one-dimensional counting technique is employed for its approximate analysis.

A second important approximation is the linearization of the equations describing average particle size. Although higher order approximations are possible within the general formulation presented here, we adopted the linearization approximation for the sake of clarity. It should however be noted that the magnitude of the parameter $\alpha$ depends strongly on the linearization, and is significantly overestimated. This follows from the fact that $\alpha$ is obtained here by fitting a straight line to the data points.

The present model is strictly valid for low degrees of filler content where different aggregates do not percolate through the system. In polymer theory, the physical picture resembles the problem of chain branching without gelation. ${ }^{[27,28]}$ However, results of the present analysis depart significantly from that of the hyperbranched polymer growth theory in the absence of excluded volume. According to this model, the distribution $W(n)$ of cluster size $n$ is given by the expression

$$
W(n)=\frac{[(f-1) n] !}{n ![(f-2) n+1] !} p^{n-1}(1-p)^{(f-2) n+1}
$$

where, $p$ and $f$ are the parameters of the theory. ${ }^{[27,28]}$ The maximum of Equation (4) is always at $n=0$, in disagreement with Equation (A15) and experimental observation.

The present model is based on several assumptions that do not hold strictly for the hydrophilic silica and PDMS used as the experimental system to check the model. For example, the individual particles are not spherical and of uniform size. However, the AFM images, such as the ones shown in Figure 2, and the aggregate size distributions obtained from them as shown in Figure 3 indicate that in the lowest filler content the dispersion in aggregate size is significantly lower and their sizes are approximately spherical. The "individual particle size" of the model corresponds to the size of an equivalent sphere obtained at infinite dilution. According to the model, this diameter is about $40 \mathrm{~nm}$, as seen from the $y$-intercept of Figure 5. This diameter may contain several individual silica particles and the immobilized layer of PDMS chains around them. The deviations of real systems from the assumptions of the model become more significant at high filler content. In this respect, the present theory is strictly applicable for low silica content.

Despite the weaknesses and approximations described in the preceding paragraphs, the proposed model (i) predicts the increase in cluster size with increasing amount of filler, (ii) explains the increase in the dispersion of aggregate sizes with increasing amount of filler, and (iii) the skewness of the distribution functions that is observed in experimental results. 


\section{Appendix}

In this section, we give the detailed derivation of Equation (1) and (3) of the text, which express the cluster size distribution and the mean cluster size of filler particles, respectively.

We use Figure 1 as a reference. The axis on which projections are made is the interval $(0, L)$. The projection of each individual particle on this axis forms a sub-interval $(0$, $D$ ) of length $D$. Let us choose two such projections for which the left-hand end (LHE) of the second projection falls on the interval $(0, D)$ of the first projection. This is equivalent to the condition that the corresponding two filler particles are either in contact or belong to the same cluster. As a result of the random placement assumption, the distribution of the LHE points of the projections is uniform in the interval $(0, L)$. Therefore, the probability of an LHE of a projection to be in a given interval $(0, D)$ is $D / L$. Since particles belong to the same cluster when their projections overlap, the number of overlapping projections is equal to the number of particles in a cluster. Again, as the result of randomness assumption, the distribution of the number of LHEs of projections in a given interval $(0, D)$ is binomial. If there are $N$ individual particles within the volume of the cylinder, then the average number $a_{0}$ of LHEs falling on the given interval $(0, D)$ is

$$
a_{0} \equiv N D / L
$$

Here, the subscript zero indicates that there are no interactions between the particles, and the random conditions hold. Interparticle interactions are defined here as either favorable where the interaction energy between the particles is negative, and the particles tend to form larger clusters, or unfavorable, where the interaction energy is positive and the particles tend to remain as far apart as possible. The volume fraction $v$ of fillers may be approximated by the ratio of the filler particles in the cylinder to the volume of the cylinder. Thus

$$
v=\frac{N \frac{\pi}{6} D^{3}}{\frac{\pi}{4} \bar{D}_{\mathrm{c}}^{2} L}
$$

Solving this expression for $N D / L$ and substituting in Equation (A1), the variable $a_{0}$ may be expressed in terms of the volume fraction of particles $v$, and the particle and cylinder diameters as

$$
a_{0}=\frac{3}{2}\left(\frac{\overline{D_{\mathrm{c}}}}{D}\right)_{0}^{2} v
$$

The subscript zero in Equation (A3) indicates the absence of interparticle interactions. Since $N$ is large, $D / L$ is small, and $a_{0}$ is finite, the probability $P_{0}(n)$ of the number $n$ of overlaps on a given interval $(0, D)$ in the absence of interparticle interactions is represented by the binomial distribution. The latter may be approximated by the Poisson distribution with mean $a_{0}$

$$
P_{0}(n)=\frac{\mathrm{e}^{-a_{0}} a_{0}^{n}}{n !}, \quad n=0,1,2, \ldots
$$

The probability $P_{0}(0)$ that no overlap occurs in $(0, D)$ is $P(0)=\mathrm{e}^{-a_{0}}$. The probability that at least one overlap occurs is $1-P(0)$ and from Equation (A4) it is obtained as $1-\mathrm{e}^{-a_{0}}$.

For the case of interactions between particles, the distribution $P_{0}(n)$ has to be modified, and the new distribution $P(n)$ must have a term reflecting the degree of interparticle interaction.

There are several possible ways of introducing the modifications in the probability function $P_{0}(n)$ due to interparticle interactions. In the presence of assumption 3 stated above, an efficient route which leads to a simple solution is to use the grand canonical ensemble representation. Thus, $P(n)$ may be expressed in the form

$$
P(n)=\frac{Q(n) \mathrm{e}^{n \mu / k T}}{\Xi}, \quad n=0,1,2, \ldots
$$

where $Q(n)$ and $\Xi$ are analogous to the canonical partition function for a system containing $n$ particles, and the grand canonical partition function, respectively. In the absence of interactions, $P(n)$ reduces to $P_{0}(n)$ and $\frac{Q(n)}{\Xi} \propto \frac{\mathrm{e}^{-a_{0}} a_{0}^{n}}{n !}$. Substituting this expression in Equation (A5), we obtain

$$
P(n) \propto \frac{\mathrm{e}^{-a_{0}} a_{0}^{n} \mathrm{e}^{\frac{n \mu}{T T}}}{n !}
$$

For particles in equilibrium where assumption 3 stated in the Theory and the Model, is valid, the chemical potential $\mu$ is the same for clusters of all sizes. ${ }^{[29]}$ This condition may be expressed as

$$
\mu=\mu_{n}=\mu_{n}^{0}+\frac{k T}{n} \ln \left(\frac{X_{n}}{n}\right)=\text { constant }, n=1,2, \ldots
$$

where, $\mu_{n}$ is the chemical potential of a cluster of size $n, X_{n}$ is its mole fraction and $\mu_{n}^{0}$ is the standard part of the chemical potential expressed as the mean interaction free energy per molecule in a cluster of size $n$. The mole fraction $X_{n}$ is related to the probability $P(n)$ by the expression $P(n)=X_{n} / \sum X_{n}$. Equation (A7) may be written as ${ }^{[25]}$

$$
X_{n} \propto n\left\{X_{1} \mathrm{e}^{\left(\mu_{1}^{0}-\mu_{n}^{0}\right) / k T}\right\}^{n}
$$

For spherical particles

$$
\mu_{n}^{0}=\mu_{\infty}^{0}+\frac{\alpha k T}{n^{1 / 3}}
$$

where $\mu_{\infty}^{0}$ is the bulk energy per molecule and

$$
\alpha=\frac{4 \pi r^{2} \gamma}{k T}
$$


where $\gamma$ is the interfacial energy per unit area and $r$ is the effective radius of a particle. Substituting Equation (A9) and (A10) into Equation (A8) and rearranging leads to

$$
X_{n}=C_{n}\left\{X_{1} \mathrm{e}^{\alpha}\right\}^{n}
$$

where the approximation $\mathrm{e}^{\alpha[1-(1 / 3)]} \approx \mathrm{e}^{\alpha}$ has been adopted.

In terms of probabilities, Equation (A11) may be written as

$$
P(n)=C_{n}\left\{P(1) \mathrm{e}^{\alpha}\right\}^{n}
$$

where $C_{n}$ is to be chosen such that $\sum_{n=0}^{\infty} P(n)=1$. Using Equation (A4) for $P(1)$ in Equation (A12), $C_{n}$ is obtained as

$$
C_{n}=\frac{1}{n ! \mathrm{e}^{a_{0} \mathrm{e}^{\alpha}}}
$$

The probability function given by Equation (A4) in the absence of interactions is now obtained, in the presence of interactions, as

$$
P(n)=\frac{\mathrm{e}^{-a_{0} \mathrm{e}^{\alpha}+\alpha n} a_{0}^{n}}{n !}
$$

Employing Stirling's approximation for the factorial in the denominator, Equation (A14) may be written in computationally more suitable form as

$$
P(n)=\frac{\mathrm{e}^{-a_{0} \mathrm{e}^{\alpha}}}{\sqrt{2 \pi}}\left[\frac{\mathrm{e}^{(\alpha+1) n}\left(\frac{a_{0}}{n}\right)^{n}}{\sqrt{n}}\right]
$$

Equation (A15) defines the distribution of cluster sizes in the filled elastomer, and is given in the text as Equation (1). Since each cluster of segments in $(0, L)$ has a unique rightmost member (see Figure 1), the number of clusters along the axis is equal to the number of their rightmost members. The condition that a projection is the rightmost member of a cluster is equivalent to the condition that no other projection has its LHE on it. The probability of this condition from Equation (A14) is $P(0)=\mathrm{e}^{-a_{0} \mathrm{e}^{\alpha}}$. From this probability relationship, we see that the parameter $a$ that is obtained in the presence of interparticle interactions is related to $\mathrm{a}_{0}$ as $a=a_{0} e^{\alpha}$. The mean number of clusters $\bar{N}_{\mathrm{c}} \equiv N P(0)$ may now be written as

$$
\bar{N}_{c}=N P(0)=N \mathrm{e}^{-a_{0} \mathrm{e}^{\alpha}}
$$

In the presence of favorable interactions between particles, Equation (A16) shows a significant decrease in the number of clusters.

For compact arrangement of the particles in the clusters, one obtains the average cluster diameter $\bar{D}_{c}$ as

$$
\frac{\bar{D}_{c}}{D}=\mathrm{e}^{\frac{1}{3} a_{0} \mathrm{e}^{\alpha}}
$$

Substituting for $\mathrm{a}_{0}$ on the right-hand side of Equation (A17) from Equation (A3) leads to

$$
\frac{\bar{D}_{c}}{D}=\mathrm{e}^{\frac{1}{2}\left(\frac{\bar{D}_{c}}{D}\right)_{0}^{2} \mathrm{e}^{\alpha} v}
$$

Expanding the right-hand side of Equation (A18), and keeping only the term linear in $\mathrm{e}^{\alpha} v$ leads to

$$
\frac{\bar{D}_{c}}{D}=1+\frac{1}{2}\left(\frac{\bar{D}_{c}}{D}\right)_{0}^{2} \mathrm{e}^{\alpha} v
$$

In the absence of interactions, $\alpha=0$ and $\frac{\overline{D_{c}}}{D}=\left(\frac{\overline{D_{c}}}{D}\right)_{0}$, Equation (A19) may be solved for $\left(\frac{\overline{D_{c}}}{D}\right)_{0}$ as

$$
\left(\frac{\bar{D}_{c}}{D}\right)_{0}=\frac{1-\sqrt{1-2 v}}{v}=1+\frac{1}{2} v
$$

where the second equality indicates the first order approximation which should be valid at low filler content. Substituting this expression in Equation (A19) leads to

$$
\frac{\bar{D}_{c}}{D}=1+\frac{1}{2} \mathrm{e}^{\alpha}\left(v+v^{2}+\frac{1}{4} v^{3}\right)
$$

which at low values of $v$ reduces to

$$
\frac{\bar{D}_{c}}{D}=1+\frac{1}{2} \mathrm{e}^{\alpha} v
$$

The relationship given by Equation (A22) is based on Equation (A17) which is valid if the particles are densely packed in a cluster and that there are no voids. In the presence of voids, the front factor $1 / 2$ in Equation (A22) is expected to be larger. In the following paragraphs, we derive the analog of Equation (A22) without the dense packing assumption.

Let the positions of the LHE's of the projections in a cluster be denoted by $x_{1}, x_{2}, \ldots, x_{n}$. The distance $x$ from $x_{1}$ to $x_{2}$ has a geometric distribution which may be approximated by the exponential distribution. The probability $p$ that $x_{2}$ will be in the interval $\left(x_{1}, x_{1}+D\right)$ is

$$
p=\int_{0}^{D} \lambda \mathrm{e}^{-\lambda x} \mathrm{~d} x=1-\mathrm{e}^{-\lambda D}
$$

where, $\lambda$ is the parameter of the distribution. But this probability is equal to the probability of having at least one $x_{i}$ in the interval $\left(x_{1}, x_{1}+D\right)$, and from the discussion of the preceding paragraphs, this is $1-\mathrm{e}^{-a}$. Therefore, $\lambda=a / D$.

The number $k$ of successive overlaps until there is no overlap is given by the geometric distribution $(1-p) p^{k}$ where $p$ is given by Equation (A6) above. The mean $E(k)$ of the successive overlaps is given as

$$
E(k)=\frac{p}{1-p}=\mathrm{e}^{a}-1
$$

If a cluster has $k$ overlapping segments, the total length of the cluster is the length $D$ of the rightmost segment plus the $k-1$ random distances between the LHE of any segment and the LHE of the next segment to its right. These distances are distributed exponentially, subject to 
the condition that each should be less than $D$ so that they are overlapping segments. This conditional distribution is

$$
p(x \mid 0<x<D)=\frac{\lambda \mathrm{e}^{-\lambda x}}{1-\mathrm{e}^{-\lambda D}}
$$

The mean $E(x)$ of this distribution is

$$
E(x)=\int_{0}^{D} x \frac{\lambda \mathrm{e}^{-\lambda x}}{1-\mathrm{e}^{-\lambda D}} \mathrm{~d} x=\frac{1}{\lambda}-\frac{D}{\mathrm{e}^{\lambda D}-1}
$$

The sum $S$ of the $k-1$ random distances is also a random variable. Its mean value $E(S)$ is given as the product of the mean of $k$ and the mean of $x$

$E(S)=E(k) E(x)=\left(\mathrm{e}^{a}-1\right)\left(\frac{1}{\lambda}-\frac{D}{\mathrm{e}^{a}-1}\right)=\frac{e^{a}-1}{\lambda}-D$

Adding the length $D$ of the last segment, the mean cluster size $\bar{D}_{\mathrm{c}}$ is obtained as

$$
\bar{D}_{\mathrm{c}}=\frac{\mathrm{e}^{a}-1}{a} D
$$

where the equality $\lambda=a / D$ is used. Solving Equation (A28) for $\bar{D}_{\mathrm{c}} / D$, expanding the right-hand side to the first order in $a$ and using Equation (A3) and the equality $a=a_{0} \mathrm{e}^{\alpha}$ leads to

$$
\frac{\bar{D}_{\mathrm{c}}}{D}=1+\frac{3}{4}\left(\frac{\bar{D}_{\mathrm{c}}}{D}\right)_{0}^{2} e^{\alpha} v
$$

In the absence of interactions, we have

$$
\left(\frac{\overline{D_{\mathrm{c}}}}{\bar{D}}\right)_{0}=1+\frac{3}{4}\left(\frac{\overline{D_{\mathrm{c}}}}{\bar{D}}\right)_{0}^{2} v
$$

Solution of this equation for $\left(\bar{D}_{\mathrm{c}} / D\right)_{0}$ leads to

$$
\left(\frac{\overline{D_{\mathrm{c}}}}{D}\right)_{0}=\frac{2}{3 v}(1-\sqrt{1-3 v})
$$

Expanding Equation (A31) into Taylor's series and keeping the first order term in $v$ leads to

$$
\left(\frac{\overline{D_{\mathrm{c}}}}{D}\right)_{0}=1+\frac{3}{4} v
$$

It is to be noted that $\left(\bar{D}_{\mathrm{c}} / D\right)_{0}$ obtained by Equation (A20)(A32) are different because the former was obtained under the assumption of compact arrangement of particles in a cluster whereas the latter was not.

Substituting Equation (A32) in Equation (A29) leads, within the first-order approximation, to

$$
\frac{\bar{D}_{\mathrm{c}}}{D}=1+\frac{3}{4} \mathrm{e}^{\alpha} v
$$

Equation (A33) is the main result of the present study, and is given in the text as Equation (3). The factor of 1/2 that appears in Equation (A22) is now given, more rigorously, as $3 / 4$.

Acknowledgements: The authors gratefully acknowledge comments by Dr. Canan Baysal which are incorporated into the final version of the manuscript. In addition, special thanks to Professor Christopher W. Macosko and Professor Mirta I. Aranguren for their helpful suggestions on the manuscript.

[1] B. Erman, J. E. Mark, "Structures and Properties of Rubberlike Networks", $1^{\text {st }}$ edition, Oxford University Press, New York 1997.

[2] [2a] B. Wang, G. L. Wilkes, J. C. Hedrick, S. C. Liptak, J. E. McGrath, Macromolecules 1991, 24, 3449; [2b] L. L. Beeccroft, C. K. Ober, Chem. Mater. 1997, 9, 1302; [2c] M. M. Demir, M. Memesa, P. Castignolles, G. Wegner, Macromol. Rapid Commun. 2006, 27, 763.

[3] R. Clement, "Hybrid Organic Inorganic Composites", J. E. Mark, Ed., American Chemical Society, Washington DC 1995, p. 29.

[4] J. E. Mark, J. Phys. Chem. B 2003, 107, 903.

[5] [5a] W. Q. Yuan, J. E. Mark, Macromol. Chem. Phys. 1999, 200, 206; [5b] M. M. Demir, Y. Z. Menceloglu, B. Erman, Polymer 2005, 46, 4127.

[6] L. Bokobza, G. Gilles, J. E. Mark, J. M. Jethmalani, E. E. Seabolt, W. T. Ford, Chem. Mater. 2002, 14, 162.

[7] W. M. Litvinov, H. Barthel, J. Weis, Macromolecules 2002, 35, 4356.

[8] M. I. Aranguren, E. Mora, C. W. Macosko, J. Colloid. Interface Sci. 1997, 195, 329.

[9] J. Berriot, F. Lequeux, L. Monnerie, H. Montes, D. Long, P. Sotta, J. Non-Cryst. Solids 2002, 719, 307.

[10] J. W. Brinke, W. M. Litvinov, J. E. G. J. Winjhoven, J. W. M. Noordermer, Macromolecules 2002, 35, 10026.

[11] M. Gussoni, F. Greco, M. Mapelli, A. Vezzoli, E. Ranucci, P. Ferruti, L. Zetta, Macromolecules 2002, 35, 1722.

[12] T. Cosgrove, C. Roberts, T. Garasanin, G. R. Schmidt, V. G. Gordon, Langmuir 2002, 18, 10080.

[13] P. Levresse, D. L. Feke, I. M. Zloczower, Polymer 1998, 39 , 3919.

[14] J. P. Cohen-Addad, P. Huchot, P. Jost, A. Pouchelon, Polymer 1989, 30, 143.

[15] J. P. Cohen-Addad, R. Ebengou, Polymer 1992, 33, 379.

[16] O. Girard, J. P. Cohen-Addad, Polymer 1991, 32, 860.

[17] D. Gurovich, C. W. Macosko, M. Tirell, Rubber Chem. Technol. 2003, 76, 1

[18] D. Gurovich, C. W. Macosko, M. Tirell, Rubber Chem. Technol. 2003, 76, 13 .

[19] G. Bushell, Ph. D. Dissertation, The University of New South Wales, 1998, Australia.

[20] J. E. Martin, J. P. Wilcoxon, D. Schaefer, J. Odinek, Phys. Rev. A 1990, 41, 4379.

[21] S. Simovic, C. A. Prestidge, Langmuir 2003, 19, 8364.

[22] V. N. Manoharan, M. T. Elsesser, D. J. Pine, Science 2003, $301,483$.

[23] M. Klüppel, G. Heinrich, Rubber Chem. Technol. 1995, 68, 623. 
[24] M. Klüppel, R. H. Schuster, G. Heinrich, Rubber Chem. Technol. 1997, 70, 243.

[25] J. E. Mark, B. Erman, "Rubberlike Elasticity: A Molecular Primer", Wiley, New York 1988.

[26] W. J. Ewens, G. R. Grant, "Statistical Methods in Bioinformatics: An Introduction", Springer, New York 2001, Chapter 5.
[27] P. J. Flory, "Principles of Polymer Chemistry", Cornell University Press, Ithaca, New York 1953.

[28] M. Rubinstein, R. H. Colby, "Polymer Physics", Oxford University Press, Oxford 2003.

[29] J. N. Israelachvili, "Intermolecular and Surface Forces", Academic Press, London 1991. 